Publ. RIMS, Kyoto Univ。

22 (1986), 57-80

\title{
Jensen Measures and Maximal Functions of Uniform Algebras
}

\author{
Dedicated to Professor Yukio Kusunoki on his 60th birthday
}

By

Cho-ichiro MATSUOKA*

\begin{abstract}
Our purpose here is to seek on an arbitrary uniform algebra the class of representing measures which admit a certain maximal function for each log-envelope function defined on the maximal ideal space of the algebra. These maximal functions can be considered as a proper generalization of those that are associated with two-dimensional Brownian motion in the concrete algebras $\boldsymbol{R}(K)$.

Most of the results already obtained from the probabilistic approach, e. g. BurkholderGundy-Silverstein inequalities, a weaker form of Fefferman's duality theorem etc., are valid for our maximal functions. The remarkable feature of our class of representing measures is that it is stable under the weak-star limit and the convex combination.

In the concrete algebras $\boldsymbol{R}(K)$, if the harmonic measure and the Keldysh measure for a given point of $K$ are different, then our class of representing measures that are supported on the topological boundary of $K$ forms an infinite-dimensional weak-star compact convex set in the dual of $\mathbb{C}(K)$.
\end{abstract}

\section{$\S 0 . \quad$ Introduction}

It is well-known that Hardy spaces on the unit disk carry the maximal functions of several types. The probabilistic approach to the analysis of them has been developed by many authors. Of course, most of the results are valid for more general Hardy spaces, if we concentrate our attention on the Brownian maximal function. The purpose here is to study these results from the viewpoint of general uniform algebra theory. That is, we shall investigate a certain class of representing measures associated with uniform algebras whose Hardy spaces admit the maximal function analogous to that of the Brownian motion.

In order to explain our strategy, let us consider the concrete algebras. We denote by $K$ a nonempty compact subset of the complex plane. $\mathbb{R}(K)$ is the uniform closure in $\boldsymbol{C}(K)$ of all rational functions with poles off $K$. We use

Communicated by S. Nakano, June 17, 1985. Revised September 2, 1985.

* Department of Mechanical Engineering, Doshisha University, Kyoto 602, Japan. 
the symbol $\lambda_{K}$ to denote the Keldysh measure associated with the set $K$ and a fixed point $p_{0}$ of $K$. By the result of A. Debiard and B. Gaveau [3], $\lambda_{K}$ is maximum in the logarithmic order among all Jensen measures for $p_{0}$ with respect to $\boldsymbol{R}(K)$. Here the logarithmic order $(\prec)$ implies the partial order over positive measures on $K$ induced by the cone of all continuous functions that are subharmonic in neighbourhoods of $K$. The same is true for each $\boldsymbol{R}(K)$-convex compact subset $F$ of $K$, if it contains $p_{0}$. Consequently, to the Keldysh measure $\lambda_{K}$ there corresponds a family $\left\{\lambda_{F}\right\}$ of Jensen measures indexed by the $\boldsymbol{R}(K)$ convex sets such that

(1) each $\lambda_{F}$ is a Jensen measure for $p_{0}$ supported on $F \ni p_{0}$,

(2) if $F \subset G$, then $\lambda_{F}<\lambda_{G}$,

(3) each $\lambda_{F}$ is maximal in the logarithmic order among all positive measures on $F$.

It is well-known that these measures are derived from two-dimensional Brownian motion starting at $p_{0}$, and accordingly Brownian maximal functions, or more precisely their conditional expectations, are defined in $L^{1}\left(\lambda_{K}\right)$ for functions $|f|^{p}$, $|u|^{p}$, where $f \in \boldsymbol{R}(K) u=\operatorname{Re} f$ and $0<p<\infty$. Furthermore, most of the results obtained in the case of the unit disk are still valid in such circumstances, if we interpret the argument on the duality $\left\langle H_{0}^{1}, L^{\infty} / H^{\infty}\right\rangle$ suitably. We shall show them from the viewpoint of general uniform algebra theory. Namely, suppose a uniform algebra $\boldsymbol{A}$ has a Jensen measure $\lambda_{\Omega}$ for which there exists a family of measures satisfying (1), (2), (3) on the maximal ideal space $\Omega$ of $A$. Here the logarithmic order is defined by using the cone of all continuous log-envelope functions on $\Omega$. Applying this assumption only, we shall establish the following:

(4) the generalized (conditional expectations of) Brownian maximal functions can be defined in $L^{1}\left(\lambda_{2}\right)$ for functions as stated above,

(5) the maximal function, together with the original function, enjoys BurkholderGundy-Silverstein inequalities,

(6) a weaker form of Fefferman's duality theorem holds, i. e. as the functionals on $H_{p_{0}}^{1}\left(\lambda_{\Omega}\right)$, abstract harmonic functions on $\Omega$ have the norm bounded by the constant times the Garsia norm of them. (Theorem 5.5, Corollary 5.6 and Theorem 5.7.)

From applicational point of view, conditions (1), (2), (3) are too hard. So we shall relax them in Definition 4.1. The relaxed conditions have the remarkable feature. That is, the class of representing measures satisfying these conditions are stable under the weak-star limit and the convex combination (Theorem 4.8, 4.9.)

In Section 6, our concern will return to the algebra $\mathbb{R}(K)$. If $K$ has an interior point $p_{0}$, and if Jensen measures for $p_{0}$ that are supported on $\partial K$ are not unique, then infinitely many Jensen measures for $p_{0}$ carried on $\partial K$ admit 
families of measures satisfying the relaxed conditions. Owing to the above stability theorems and Remark 6.6, they form an infinite dimensional weak-star compact convex set in the dual of $C(K)$. Of course, all Jensen measures cited here satisfy the results stated above.

Finally, the author expresses his deep gratitude to Professor T. Gamelin who advised him on all the phases of this article. The author's thanks are also due to the referee for the valuable communication.

\section{$\S 1$. Preliminaries}

Throughout this paper, we follow the useful terminologies in T. W. Gamelin [5], [6]. We first clarify the notations, and mention some of the basic facts without proofs. Details can be found in [5], [6].

In the sequel, $\boldsymbol{A}$ always denotes an arbitrary, but fixed, uniform algebra on some compact Hausdorff space. The letter $\Omega$ will designate the maximal ideal space of $\boldsymbol{A}$. Let $h$ be a real-valued function defined on a subset $E$ of $\Omega$. The lower log-envelope $\check{h}$ of $h$ is an extended real-valued function on $\Omega$ defined as

$$
\check{h}=\sup \{c \log |f|: f \in \boldsymbol{A}, c \in \mathbb{R}, c \geqq 0, h \geqq c \log |f| \text { on } E\} \text {. }
$$

We use the letter $\boldsymbol{J}$ to denote the totality of functions $f$ in $\mathbb{C}_{R}(\Omega)$ such that $\check{f}=f$ on $\Omega$. Clearly $J$ is stable in the $\max$ operation $\vee$, i. e. $f \vee g=\max \{f, g\}$ is contained in $\boldsymbol{J}$ provided $f, g$ belong to $\boldsymbol{J}$. Therefore, $\boldsymbol{J}-\boldsymbol{J}$ is uniformly dense in $C_{R}(\Omega)$, because $J$ contains $\operatorname{Re} A$, the real parts of functions in $A$.

Since $\boldsymbol{J}$ is a convex cone of $C_{R}(\Omega)$, it defines a partial order over all finite regular Borel measures that are supported on $\Omega$. We are interested in the order restricted within the positive measures on $\Omega$. This order relation will be denoted by the symbol $\prec$ and called the logarithmic order simply. Here we note that a probability measure $\mu$ is a Jensen measure for $p \in \Omega$ if and only if it satisfies the relation $\delta_{p} \prec \mu$, where $\delta_{p}$ is the Dirac measure at $p \in \Omega$.

We say that a positive measure is maximal if it is a maximal element in the logarithmic order among all positive measures on $\Omega$. It is known that every positive measure is dominated by a maximal positive measure concerning this order relation. Also it is known that a positive $\mu$ is maximal if and only if it satisfies $\check{h}=h$ a. e. $\mu$ for all $h$ of $C_{R}(\Omega)$. In this characterization, if $\mu$ is supported on a closed set containing the Shilov boundary of $A, h$ can be replaced by continuous functions defined on the closed set.

Let $p$ be a point on $\Omega$ for which the point mass $\delta_{p}$ is maximal in the logarithmic order. The totality of such points is known as the Jensen boundary of $A$. It is a dense subset of the Shilov boundary of $A$, and contains all generalized peak points with respect to $A$. In case that $A$ is separable, all maximal positive measures are supported on the Jensen boundary, which is a $G_{\tilde{o}^{-}}$-subset of $\Omega$ in this case. 
Let $E$ be a nonempty compact subset of $\Omega$. The $A$-convex hull of $E$ is the totality of points in $\Omega$ whose evaluation functionals $A \ni f \mapsto f(p)$ satisfy the inequality

$$
|f(p)| \leqq\|f\|_{E}=\sup \{\mid f(q) !: q \in E\} \quad \text { for all } f \text { of } \boldsymbol{A} .
$$

Let $A_{E}$ denote the closure in $\boldsymbol{C}(E)$ of the restriction algebra $\left.\boldsymbol{A}\right|_{E}$. Then the maximal ideal space of $\boldsymbol{A}_{E}$ is identical with the $\boldsymbol{A}$-convex hull of $E$. Therefore all the facts quoted above are valid for them. In this case, we note that maximal positive measures associated with $\boldsymbol{A}_{E}$ are supported on $E$, because $E$ contains the Shilov boundary of $\boldsymbol{A}_{E}$. We say that a positive measure is maximal on $E$ if it is supported on $E$ and maximal in the logarithmic order with respect to $\boldsymbol{A}_{E}$. Since $\boldsymbol{A}_{E}$ is dense in $\boldsymbol{A}_{E}$, a positive $\mu$ is maximal on $E$ if and only if it satisfies $\check{h}=h$ a. e. $\mu$ for all $h \in C_{R}(E)$.

We are now in a position to define a subfamily of Jensen measures as stated in the preceding section. Recall that this subfamily has been desired to be as small as possible. For a given point $q \in \Omega$, put $G=\Omega[g \leqq r]=$ $\{p \in \Omega: g(p) \leqq r\}$, where $g \in J$ and $r>g(q)$. Clearly $G$ is $A$-convex. We denote by $\mathscr{F}_{q}$ the set of all such $G$ 's.

Definition 1.1. Let $\left\{\lambda_{G}: G \in \mathscr{F}_{q}\right\}$ be a family of Jensen measures indexed by $\mathscr{I}_{q}$. We say that this family is maximally consistent if it satisfies

(1) each element $\lambda_{G}$ is a Jensen measure for $q$ supported on $G$,

(2) for $G, K$ of $\mathscr{F}_{q}$, if $G \subset K$, then $\lambda_{G}<\lambda_{K}$,

(3) each $\lambda_{G}$ is maximal on $G$ in the logarithmic order.

The terminal measure $\lambda_{\Omega}$ of the family will be called a Keldysh measure for $q$.

\section{$\S 2$. Some Properties of Locally Maximal Measures}

The aim in this section is to prove Theorem 2.4. In comparison with the probabilistic theory of Hardy spaces, it seems that this theorem corresponds to the strong Markov property of Brownian motion.

The powerful device for our investigation is the following deep result due to T. Gamelin and N. Sibony ([7] cf. [6]).

Theorem 2.1. (The localization principle for the Jensen boundary) Let $\mathbb{B}$ be a uniform algebra and let $K$ be a closed subset of the maximal ideal space of $\boldsymbol{B}$. Suppose an interior point $p$ of $K$ belongs to the Jensen boundary of $\boldsymbol{B}_{K}$. Then $p$ belongs to the Jensen bonndary of $\boldsymbol{B}$.

Applying this theorem, we first establish a localization theorem for locally maximal measures. Here we note that the next theorem is contained in the above result if a uniform algebra in problem is separable. 
Theorem 2.2. Let $\boldsymbol{A}$ be a uniform algebra with the maximal ideal space $\Omega$, and let $K \subset \Omega$ be compact. Suppose a positive measure $\mu$ is supported on Int $K$ and maximal on $K$. Then it is also maximal on $\Omega$.

Proof. At first given a countable subset $S$ of $A$, we construct a separable subalgebra $\mathbb{B}$ of $\boldsymbol{A}$ which contains $S$ and has several nice properties.

Let $\left\{V_{n}\right\}_{1}^{\infty}$ be a sequence of open subsets of Int $K$ such that each $V_{n}$ is defined by a finite number of functions $\left\{f_{\imath}^{n}\right\}$ in $A$, i. e. $V_{n}=\bigcap_{2} \Omega\left[\left|f_{\imath}^{n}\right|<1\right]$. Since $\mu$ is regular, we can take $\left\{V_{n}\right\}$ so that $\mu\left\{\bigcup V_{n}\right\}=\mu(\operatorname{Int} K)=\|\mu\|$. Let denote by $\boldsymbol{B}_{1}$ the closed subalgebra generated by $S$, constants and all $f_{2}^{n}$. Clearly $\mathbb{B}_{1}$ is separable. By induction we manufacture a sequence $\left\{\boldsymbol{B}_{n}\right\}_{n=1}^{\infty}$ of separable closed subalgebra in $A$ so that they satisfy

(1) $\boldsymbol{B}_{n} \subset \boldsymbol{B}_{n+1} \quad(n \in \mathbb{N})$,

(2) if $\tau$ is a nontrivial character (multiplicative linear form) on $\mathbb{B}_{n+1}$, then $\left.\tau\right|_{B_{n}}$ coincides with the evaluation functional of some point in $\Omega$,

(3) if $D_{n}$ is the closed subalgebra of $C_{R}(\Omega)$ generated by $\operatorname{Re} \boldsymbol{B}_{n}$, then for each $h$ of $D_{n}$ and $\varepsilon>0$, there are functions $\left\{g_{\jmath}\right\}$ of $\mathbb{B}_{n+1}$ and $c_{\jmath} \geqq 0$ such that

$$
h \geqq c_{\jmath} \log \left|g_{\jmath}\right| \quad \text { on } K, \quad \int \max \left\{c_{\jmath} \log \left|g_{\jmath}\right|\right\} \mathrm{d} \mu>\int h \mathrm{~d} \mu-\varepsilon .
$$

Assume that $\mathbb{B}_{1} \cdots \boldsymbol{B}_{n}$ have already been constructed. Since $D_{n}$ in (3) is separable by induction hypothesis, it has a countable dense subset $\left\{h_{1}, h_{2}, \cdots\right\}$. Then for each $h_{\jmath}$ and $m \in \mathbb{N}$ we can find a finite number of functions $\left\{g_{\jmath m}^{k}\right\}$ in $A$ and $c^{k} \geqq 0$ such that $h_{\jmath} \geqq c^{k} \log \left|g_{\jmath m}^{k}\right|$ on $K, \int \max _{k}\left\{c^{k} \log \left|g_{\jmath m}^{k}\right|\right\} \mathrm{d} \mu>\int h_{\jmath} \mathrm{d} \mu-1 / m$. These are possible, because $\mu$ is maximal on $K$ in the logarithmic order. Let denote by $\widetilde{\mathbb{B}}_{n}$ the closed subalgebra of $\boldsymbol{A}$ generated by $\mathbb{B}_{n}$ and all $g_{j m}^{k}$. Clearly $\widetilde{\mathbb{B}}_{n}$ is separable. Moreover, any closed subalgebra of $A$ containing $\widetilde{\mathbb{B}}_{n}$ satisfies condition (3) automatically. Let $i: \widetilde{B}_{n} \subset \mathbb{A}$ be the canonical inclusion map and $i^{*}: \mathbb{A}^{*} \rightarrow \widetilde{B}_{n}^{*}$ be its adjoint. Since relativized weakstar topology on the maximal ideal space $\mathscr{M}\left(\widetilde{\boldsymbol{B}}_{n}\right)$ of $\widetilde{\boldsymbol{B}}_{n}$ is metrizable and $i *(\Omega)$ is compact, $\mathscr{M}\left(\widetilde{\boldsymbol{B}}_{n}\right) \backslash i^{*}(\Omega)$ is sigma compact. So the set

$$
(i *)^{-1}\left(\mathcal{M}\left(\widetilde{\boldsymbol{B}}_{n}\right) \backslash i *(\Omega)\right) \cap \dot{b} A^{*}
$$

is sigma compact also, where $b A^{*}$ is the closed unit ball of $\mathbb{A}^{*}$. Therefore we can find a countable subset $Q$ of $A$ so that for each $\tau \in b A^{*}$ with $i^{*}(\tau) \cong$ $\mathscr{M}\left(\widetilde{\mathbb{B}}_{n}\right) \backslash i^{*}(\Omega)$, there are elements $f, g$ of $Q$ satisfying $\tau(f g) \pm \tau(f) \tau(g)$. Denote by $\boldsymbol{B}_{n+1}$ the closed subalgebra of $\boldsymbol{A}$ generated by $\widetilde{\mathbb{E}}_{n}$ and $Q$.

It is now clear that $\mathbb{B}_{n+1}$ is separable and satisfies (1), (2), (3). Thus by induction we obtain the desired sequence $\left\{\mathbb{B}_{n}\right\}$ of separable closed subalgebras in $A$. Let $B$ be the closure of $\cup_{n=1}^{\infty} \mathbb{B}_{n}$. Then $\mathbb{B}$ is a closed separable subalgebra in $A$ with $S$ and constants. Denote by $i: \mathbb{B} \hookrightarrow A$ the canonical inclusion 
map, and by $i^{*}: \mathbb{A}^{*} \rightarrow \boldsymbol{B}^{*}$ its adjoint. Then $i^{*}(\Omega)$ coincides with $\mathscr{M}_{\boldsymbol{B}}$, the maximal ideal space of $\boldsymbol{B}$. Indeed, pick up any point $\tau \in \mathcal{M}_{B}$. Then by (2) $\tau \mid \mathbb{B}_{n}$ is identical with some evaluation functional. Put $P_{n}(\tau)=\left\{p \in \Omega: g(p)=g(\tau), g \in \boldsymbol{B}_{n}\right\}$. It is clear that $P_{n}(\tau)$ is compact and decreasing along the index $n$. Therefore $\bigcap P_{n}(\tau)$ is nonempty, and so $i *\left(\cap P_{n}(\tau)\right)=\tau$. This implies that $\mathscr{M}_{B}$ is identical with the compact Hausdorff space $\tilde{\Omega}$ obtained from $\Omega$ by regarding each level set $\cap P_{n}(\tau)$ as one point. (We consider so in the sequel.) Let $j: \Omega \rightarrow \widetilde{\Omega}$ be a continuous map so that $f(p)=f(j(p))$ for all $f \in B$ and $p \in \Omega$. The map $j$ gives

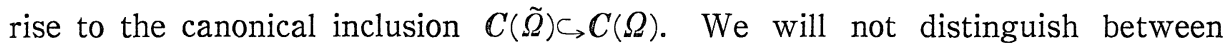
$\mathbb{C}(\tilde{\Omega})$ and its image under this inclusion. Denote by $\tilde{\mu}$ the restriction of $\mu$ onto the Baire sub $\sigma$-algebra $\sigma\{\boldsymbol{C}(\tilde{\Omega})\} . \tilde{\mu}$ can be viewed as a regular Borel measure on $\tilde{\Omega}$. We show that $\tilde{\mu}$ is maximal on $\tilde{\Omega}$ in the logarithmic order associated with $\boldsymbol{B} \subset \boldsymbol{C}(\tilde{\Omega})$.

Firstly by very definition of $\left\{V_{n}\right\}$, each $j\left(V_{n}\right)$ is open in $\tilde{\Omega}$. Therefore $\tilde{\mu}$ is supported on the interior of $j(K)$. On the other hand $\cup D_{n}$ is uniformly dense in $\boldsymbol{C}_{\boldsymbol{R}}(\tilde{\Omega})$. This implies that $\tilde{\mu}$ is maximal on $j(K)$ by (3). In particular $\tilde{\mu}$ is supported on the Jensen boundary $X_{K}$ of $\boldsymbol{B}_{\jmath(K)}$, because $X_{K}$ is $G_{\delta}$-set. $\left(\boldsymbol{B}_{3(K)}\right.$ is separable.)

By Theorem 2.1, all points in $X_{K} \cap \operatorname{Int} j(K)$ belong to the Jensen boundary of $\boldsymbol{B}$. This yields that $\tilde{\mu}$ is supported on the Jensen boundary of $\boldsymbol{B}$, and so $\tilde{\mu}$ is maximal on $\tilde{\Omega}$.

We are now in a position to complete the proof. Recall that $\boldsymbol{B}$ contains the countable subset $S$ of $\boldsymbol{A}$ previously given. Assume that the assertion is false. Then there exists a function $h$ of $C_{R}(\Omega)$ such that $\int h \mathrm{~d} \mu>\int \check{h} \mathrm{~d} \mu$. Since the subalgebra of $C_{R}(\Omega)$ generated by $\operatorname{Re} A$ is uniformly dense in $C_{R}(\Omega)$, we may assume that $h$ is in this subalgebra. Then we can find a finite subset $S$ of $\boldsymbol{A}$ so that the algebra generated by $\operatorname{Re} S$ contains the above function $h$. Let $B$ be our separable subalgebra of $A$ containing $S$. Then we can regard $h$ as a function in $\mathbb{C}_{R}(\tilde{\Omega})$. Put

$$
\tilde{h}=\sup \{c \log |g|: h \geqq c \log |g| \text { on } \Omega, c \geqq 0, g \in \boldsymbol{B}\} .
$$

Then $\int h \mathrm{~d} \mu=\int h \mathrm{~d} \tilde{\mu}=\int \tilde{h} \mathrm{~d} \tilde{\mu}=\int \tilde{h} \mathrm{~d} \mu$, because $\tilde{\mu}$ is maximal on $\tilde{\Omega}$. On the other hand $\int h \mathrm{~d} \mu>\int \breve{h} \mathrm{~d} \mu \geqq \int \tilde{h} \mathrm{~d} \mu=\int h \mathrm{~d} \mu$, a contradiction.

We need a localized form of the above theorem. Recall that an $\boldsymbol{A}$-convex compact subset $K$ of $\Omega$ can be identified with the maximal ideal space of $\mathbb{A}_{K}$.

Corollary 2.3. Let $K$ be an A-convex compact subset of $\Omega$, and let $F$ be a closed subset of $K$ with the interior $U$ relative to $K$. Suppose a positive measure $\mu$ is supported on $U$ and maximal on $F$ in the logarithmic order. Then $\mu$ is maximal on $K$. 
Theorem 2.4. Let $\mathbb{A}$ be a uniform algebra with the maximal ideal space $\Omega$, and let $F$ be a closed subset of an A-convex compact set $G \subset \Omega$. Suppose that positive measures $\mu_{F}, \mu_{G}$ satisfy the relation $\mu_{F}<\mu_{G}$, and that $\mu_{F}$ is maximal on $F$. Then for the interior $U$ of $F$ relative to $G$, the restriction measure $\mu_{F} \mid U$ is absolutely continuous with respect to $\mu_{G}$, and the density $\mathrm{d}\left(\mu_{F} \mid U\right) / \mathrm{d} \mu_{G}$ is bounded by the constant 1 .

Proof. Put $\sigma=\mu_{F} \mid U$. By Corollary 2.3, $\sigma$ is maximal on $G$ i. e. $\check{g}=g$ a. e. $\sigma$ for all $g$ of $C_{R}(G)$. For our purpose it suffices to prove that $\sigma(E) \leqq \mu_{G}(E)$ for all compact subset $E$ of $G$. Assume that $\mu_{G}(E)<\sigma(E)$ for some compact $E \subset G$. Let $g$ be a function in $\mathbb{C}_{R}(G)$ such that $g \mid E=1,0 \leqq g \leqq 1$ and $\int g \mathrm{~d} \mu_{G}<\sigma(E)$. Then by $\mu_{F}<\mu_{G}$ and $0 \leqq \check{g} \leqq g$, we are led to a contradiction

$$
\sigma(E)>\int g \mathrm{~d} \mu_{G} \geqq \int \check{g} \mathrm{~d} \mu_{G} \geqq \int \check{g} \mathrm{~d} \mu_{F} \geqq \int \check{g} \mathrm{~d} \sigma=\int g \mathrm{~d} \sigma \geqq \sigma(E) .
$$

\section{§3. Conditional Expectations Between Ordered Measures}

In order to clarify our purpose here, we first mention the result. The notations will be explained in the argument.

Theorem 3.1. Let $\mu_{1}, \mu_{2}$ be positive measures on $\Omega$ with $\mu_{1} \prec \mu_{2}$. Then there exists a positive measure $\nu$ on the product space $\Omega \times \Omega$ and a linear map $T: L^{p}\left(\mu_{2}\right)$ $\rightarrow L^{p}\left(\mu_{1}\right), 1 \leqq p \leqq \infty$, with $\|T\|_{p}=1$ as follows.

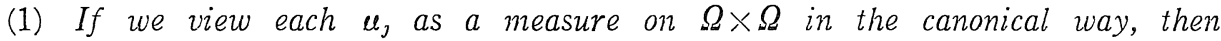
$\nu\left|\sigma\{C(\Omega) \otimes 1\}=\mu_{1}, \nu\right| \sigma\{1 \otimes C(\Omega)\}=\mu_{2}$ i.e.

$$
\int h \otimes 1 \mathrm{~d} \nu=\int h \mathrm{~d} \mu_{1}, \quad \int 1 \otimes h \mathrm{~d} \nu=\int h \mathrm{~d} \mu_{2}, \quad h \in \mathbb{C}(\Omega) .
$$

(2) $(T h) \otimes 1=E(1 \otimes h \mid \sigma\{C(\Omega) \otimes 1\})$ a. e. $\nu, \quad h \in L^{p}\left(\mu_{2}\right)$.

(3) $T g \geqq g$ a.e. $\mu_{1}, g \in J$. In particular, for every $g \in \mathbb{A}$ or $g \in \operatorname{Re} \mathbb{A}, T g=g$ a.e. $\mu_{1}$.

The map $T$ will be called a conditional expectation between ordered measures $\mu_{1}$ and $\mu_{2}$. (Note that conditional expectations can be characterized as continuous positive linear maps from $L^{p}\left(\mu_{2}\right)$ into $L^{p}\left(\mu_{1}\right)$ satisfying condition (3).)

Proof. Let $D=\left\{E_{1} \cdots E_{n}\right\}$ be the decomposition of $\Omega$ into a finite number of pairwise disjoint Borel sets. We denote by $\mathfrak{F}$ the totality of such decompositions. $\mathfrak{F}$ has the canonical order $\prec$. Namely, for any pair $D_{1}, D_{2}$ of $\mathfrak{F}$ with $D_{\jmath}=\left\{E_{\jmath 1} \cdots E_{\jmath_{j}}\right\} \quad(j=1,2)$, the relation $D_{1}<D_{2}$ implies that each member $E_{2 k}$ of $D_{2}$ is contained in some $E_{1 m}$ of $D_{1}$. Call $\mathfrak{F}_{D}=\left\{D^{\prime}: D<D^{\prime}, D^{\prime} \in \mathfrak{F}\right\}$. Then putting $D_{3}=\left\{E_{1 j} \cap E_{2 k}\right\}_{j, k}$ for $D_{1}, D_{2}$ as above, we see that $\mathfrak{\mho}_{D_{1}} \cap \mathfrak{F}_{D_{2}}=\widetilde{\mho}_{D_{D_{3}}}$. This implies that the family $\left\{\mathfrak{F}_{D}: D \in \mathfrak{F}\right\}$ forms a filter base in the power set of 
$\mathfrak{F}$ with respect to the set theoretic inclusion. Pick up an arbitrary ultra filter $\mathfrak{U}$ containing this filter base, and fix it throughout. Note that any map from $\mathfrak{\mho}$ into a compact Hausdorff space always has the limit along $\mathfrak{U}$.

Let $\mu_{1}, \mu_{2}$ be positive measures supported on $\Omega$ with $\mu_{1} \prec \mu_{2}$. For each member $D=\left\{E_{1} \cdots E_{n}\right\}$ of $\mathfrak{F}$ we consider the restriction measures $\mu_{1} ! E_{k}$ $(1 \leqq k \leqq n)$. By Cartier-Fell-Meyer's theorem (cf. [1]), there exists a decomposition $\mu_{2}=\sum_{k=1}^{n} \mu_{2 k}$ of $\mu_{2}$ into nonnegative elements $\left\{\mu_{2 k}\right\}_{1}^{n}$ so that they satisfy the relation $\mu_{1} \mid E_{k} \prec \mu_{2 k}(1 \leqq k \leqq n)$. We fix one of such decompositions, $\mu_{2}=$ $\mu_{21}+\cdots+\mu_{2 n}$ for each $D$ of $\mathfrak{\wp}$.

Next, let $\Omega_{1}, \Omega_{2}$ be two copies of $\Omega$ and consider the direct product space $\Omega^{2}=\Omega_{1} \times \Omega_{2}$. Each $C\left(\Omega_{\jmath}\right)$ can be regarded as a subspace of $C\left(\Omega^{2}\right)$ in the obvious manner $(j=1,2)$. We denote them by $C\left(\Omega_{1}\right) \otimes 1$ and $1 \otimes C\left(\Omega_{2}\right)$ respectively. That is, for an $f$ of $C(\Omega),(f \otimes 1)\left(p_{1}, p_{2}\right)=f\left(p_{1}\right),(1 \otimes f)\left(p_{1}, p_{2}\right)=f\left(p_{2}\right)$. The measure $\mu_{1}$ (resp. $\mu_{2}$ ) can be regarded as a measure defined on a Baire sub $\sigma$-algebra $\sigma\left\{C\left(\Omega_{1}\right) \otimes 1\right\}$ (resp. $\sigma\left\{1 \otimes C\left(\Omega_{2}\right)\right\}$ ). We use the same notation $\mu_{1}$ (resp. $\mu_{2}$ ) to denote it.

Now, fixing a point $p_{k}$ of $E_{k}$ for each $D=\left\{E_{1} \cdots E_{n}\right\}$ of $\mathfrak{\mho}$, we define a positive measure $\nu_{D}$ on $\Omega^{2}$ by

$$
\nu_{D}=\delta_{p_{1}} \otimes \mu_{21}+\cdots+\delta_{p_{n}} \otimes \mu_{2 n}, \quad\left(\mu_{1} \mid E_{k} \prec \mu_{2 k}, 1 \leqq k \leqq n\right) .
$$

Here $\delta_{p_{j}}$ is the point mass at $p_{\jmath} \in \Omega_{1}$, and $\delta_{p_{j}} \otimes \mu_{2 j}$ denotes the product measure of $\delta_{p_{j}}$ and $\mu_{2 j}$. That is

$$
\int h(p, q) \mathrm{d}\left(\delta_{p_{\jmath}} \otimes \mu_{2 \jmath}\right)=\int h\left(p_{\jmath}, q\right) \mathrm{d} \mu_{2 \jmath}, \quad h \in C\left(\Omega^{2}\right) .
$$

The total mass $\left\|\nu_{D}\right\|$ of $\nu_{D}$ is equal to $\left\|\mu_{1}\right\|=\left\|\mu_{2}\right\|$. Therefore, the map, $D \rightarrow \nu_{D}$ from $\mathfrak{F}$ into the dual of $C\left(\Omega^{2}\right)$ is bounded, and so it has the weak* limit $\nu$ along U. It is easily seen that $\nu \mid \sigma\{1 \otimes C(\Omega)\}=\mu_{2}$. Moreover the relation $\nu \mid \sigma\{C(\Omega) \otimes 1\}$ $=\mu_{1}$ holds. Indeed, given a $g \in \mathbb{C}(\Omega)$ and $\varepsilon>0$, we take $D^{\prime}$ from $\widetilde{F}$ so that the oscillation of $g$ on each member of $D^{\prime}$ is less than $\varepsilon$. In particular, if $D=$ $\left\{E_{1} \cdots E_{n}\right\} \in \widetilde{\mho}_{D^{\prime}}$,

$$
\sup \left\{|g(p)-g(q)|: p, q \in E_{k}\right\}<\varepsilon, \quad 1 \leqq k \leqq n .
$$

So by (3.1), for every $D$ of $\widetilde{\mho}_{D^{\prime}}$,

$$
\left|\int g \otimes 1 \mathrm{~d} \nu_{D}-\int g \mathrm{~d} \mu_{1}\right|=\left|\sum_{E_{j}=D} \int_{E_{j}}\left\{g\left(p_{\jmath}\right)-g\right\} \mathrm{d} \mu_{1}\right| \leqq \varepsilon\left\|\mu_{1}\right\| .
$$

On the other hand, $\mathfrak{U}$ contains $\widetilde{\mho}_{D^{\prime}}$. Therefore taking the limit in the above, $\left|\int g \otimes 1 \mathrm{~d} \nu=\int g \mathrm{~d} \mu_{1}\right| \leqq \varepsilon\left\|\mu_{1}\right\|$. Letting $\varepsilon \rightarrow 0$, we see that $\int g \otimes 1 \mathrm{~d} \nu=\int g \mathrm{~d} \mu_{1}$. Since $g \in C(\Omega)$ is arbitrary, we conclude that $\nu \mid \sigma\{C(\Omega) \otimes 1\}=\mu_{1}$. Once these relations have been established, we need not hesitate to use the notation $L^{p}\left(\mu_{1}\right) \otimes 1$ (resp. 
$\left.1 \otimes L^{p}\left(\mu_{2}\right)\right)$ for the canonical inclusion: $L^{p}\left(\mu_{1}\right) \subset L^{p}(\nu)$ (resp. $\left.L^{p}\left(\mu_{2}\right) \subset L^{p}(\nu)\right)$, $0<p \leqq \infty$.

Finally, the conditional expectation on $L^{1}(\nu)$ with respect to $\sigma\{\mathbb{C}(\Omega) \otimes 1\}$ satisfies the relation:

$$
E(1 \otimes g \mid \sigma\{\boldsymbol{C}(\Omega) \otimes 1\}) \geqq g \otimes 1 \quad \text { a. e. } \quad \nu
$$

for every $g$ of $\boldsymbol{J}$. Indeed, given a $g$ of $\boldsymbol{J}$ and $h$ of $\mathbb{C}(\Omega)$ we define the map $D \rightarrow \tilde{g}_{D}$ from $\widetilde{\jmath}$ into $L^{\infty}\left(\mu_{1}\right)$ by

$$
\tilde{g}_{D}=\int g \mathrm{~d} \mu_{2 k} /\left\|\mu_{2 k}\right\| \quad \text { on } \quad E_{k},
$$

where $D=\left\{E_{1} \cdots E_{n}\right\}$ and $\mu_{1} \mid E_{k} \prec \mu_{2 k}(1 \leqq k \leqq n)$. Since $\left\|\tilde{g}_{D}\right\|_{\infty} \leqq\|g\|_{\infty}$, this map has the weakstar limit $\tilde{g}$ along $\mathfrak{H}$. Here we take $\widetilde{\mho}_{D^{\prime}}$ so as to satisfy (3.2) with $h$ in place of $g$. Let $D=\left\{E_{k}\right\}$ belong to $\widetilde{\mho}_{D^{\prime}}$. Then by (3.1), we see that

$$
\int h \otimes g \mathrm{~d} \nu_{D}=\int(h \otimes 1)(1 \otimes g) \mathrm{d} \nu_{D}=\sum_{E_{j} \in D} h\left(p_{j}\right) \int g \mathrm{~d} \mu_{2 j}=\sum_{E_{j} \in D} h\left(p_{j}\right) \int_{E_{j}} \tilde{g}_{D} \mathrm{~d} \mu_{1} .
$$

Therefore,

$$
\left|\int h \otimes g \mathrm{~d} \nu_{D}-\int h \tilde{g}_{D} \mathrm{~d} \mu_{1}\right| \leqq\left\|\mu_{1}\right\|\|g\|_{\infty} \sup _{j}\left\{\left\|h-h\left(p_{j}\right)\right\|_{E_{j}}\right\} \leqq \varepsilon\left\|\mu_{1}\right\|\|g\|_{\infty} .
$$

so that $\left|\int h \otimes g \mathrm{~d} \nu-\int h \tilde{g} \mathrm{~d} \mu_{1}\right| \leqq \varepsilon\left\|\mu_{1}\right\|\|g\|_{\infty}$. Letting $\varepsilon \rightarrow 0$, we have that $\int h \otimes g \mathrm{~d} \nu$ $=\int h \tilde{g} \mathrm{~d} \mu_{1}$. Since $h \in C(\Omega)$ is arbitrary, this yields

$$
\tilde{g} \otimes 1=E(1 \otimes g \mid \sigma\{C(\Omega) \otimes 1\}) \quad \text { a. e. } \quad \text {. }
$$

On the other hand, if we take $\mathfrak{F}_{D^{\prime}}$ so that it satisfies (3.2) with $g$, then for $D=\left\{E_{1} \cdots E_{n}\right\} \in \mathfrak{F}_{D^{\prime}}$,

$$
(g-\varepsilon)\left\|\mu_{2 k}\right\| \leqq \int_{E_{k}} g \mathrm{~d} \mu_{1} \leqq \int g \mathrm{~d} \mu_{2 k} \quad \text { on each } \quad E_{k} .
$$

This is due to the facts $\mu_{1} \mid E_{k}\left\langle\mu_{2 k}\right.$ and $g \in \mathscr{J}(1 \leqq k \leqq n)$. Hence $g-\varepsilon \leqq \tilde{g}_{D}$ a. e. $\mu_{1}$, and so $g-\varepsilon \leqq \tilde{g}$ a.e. $\mu_{1}$. Letting $\varepsilon \rightarrow 0$, we conclude that $\tilde{g} \geqq g$ a. e. $\mu_{1}$. Together with (3.5), this yields (3.3).

\section{$\S 4$. Maximal Functions Associated with Keldysh Measures}

From now on, we set about to construct the maximal function associated with a Keldysh measure. But our argument is applicable to more wide class of Jensen measures. So we wish to represent the results in the form valid for them.

Definition 4.1. Let $\left\{\lambda_{G}: G \subseteq \mathscr{F}_{q}\right\}$ be a family of Jensen measures indexed by $\mathscr{F}_{q}$, where $q$ is an arbitrary point of $\Omega$, and $\mathscr{I}_{q}$ is a subclass of $A$-convex sets 
introduced in Section 1. Then the family is said to be consistent if it satisfies the following conditions.

(1) Every $\lambda_{G}$ is a Jensen measure for $q$ supported on $G$.

(2) For $G, K$ of $\mathscr{F}_{q}, \lambda_{G}<\lambda_{K}$ whenever $G \subset K$.

(3) For $G, K$ of $\mathscr{F}_{q}$ with $G \subset K$, let $U$ be the interior of $G$ relative to $K$. Then the restriction measure $\lambda_{G} \mid U$ satisfies the inequality, $0 \leqq \mathrm{~d}\left(\lambda_{G} \mid U\right) / \mathrm{d} \lambda_{K} \leqq 1$ a. e. $\lambda_{K}$.

By Theorem 2.4, the next observation is now obvious.

Proposition 4.2. Every maximally consistent family of Jensen measures is consistent.

Other examples of consistent families will be presented in Section 6 .

Let $\left\{\lambda_{G}: G \in \mathscr{F}_{q}\right\}$ be a consistent family of Jensen measures with a given base point $q \in \Omega$. In the sequel, we often deal with the restriction measures $\lambda_{\Omega[g \leqq t]} \mid \Omega[g<t]$ and $\lambda_{\Omega[g \leqq t]} \mid \Omega[g=t]$, where $g \in \boldsymbol{J}$ and $t \in \boldsymbol{R}, t>g(q)$. So we adopt the following notations for convenience, i. e.

$$
\begin{aligned}
& \lambda[g \leqq t]= \begin{cases}\lambda \Omega[g \leqq t], & t>g(q) \\
\delta_{q}, & \text { otherwise }\end{cases} \\
& \lambda[g<t]= \begin{cases}\lambda[g \leqq t] \mid \Omega[g<t], & t>g(q) \\
0, & \text { otherwise }\end{cases} \\
& \lambda[g=t]= \begin{cases}\lambda[g \leqq t] \mid \Omega[g=t], & t>g(q) \\
\delta_{q}, & \text { otherwise. }\end{cases}
\end{aligned}
$$

Lemma 4.3. Let $f \in \boldsymbol{A}$ and $0<p<\infty$. Then $g=|f|^{p}$ belongs to $\boldsymbol{J}$, i.e. $\check{g}=g$. Moreover if $g \in \boldsymbol{J}$, so is the function $\exp \{g-c\}, c \in \boldsymbol{R}$.

Proof. (cf. [6]) Let $\mu$ be an arbitrary Jensen measure for a given point $\omega$ of $\Omega$. Applying Jensen's convexity inequality to $\log |f(\omega)| \leqq \int \log |f| \mathrm{d} \mu$ and $Q(x)=\exp \{p x\}$, we have that $|f|^{p}(\omega) \leqq \int|f|^{p} \mathrm{~d} \mu$. So we conclude that $|f|^{p}$ is a log-envelope function on $\Omega$. In the similar way, we have the second assertion.

Lemma 4.4. Let $\left\{\lambda_{G}: G \in \mathscr{F}_{q}\right\}$ be an arbitrary consistent family and let $g \in J$. Then for every pair $a, b \in \boldsymbol{R}$ with $a \leqq b$

$$
0 \leqq \mathrm{~d} \lambda[g<a] / \mathrm{d} \lambda_{\Omega} \leqq \mathrm{d} \lambda[g<b] / \mathrm{d} \lambda_{\Omega} \leqq 1 \text { a.e. } \lambda_{\Omega} \text {. }
$$

Proof. Note that $\Omega[g \leqq a] \subset \Omega[g \leqq b]$ and $\Omega[g<t]$ is an open subset of $\Omega$. Therefore from condition (3) of Definition $4.1,0 \leqq \mathrm{~d} \lambda[g<a] / \mathrm{d} \lambda[g<b] \leqq 1$ and 
$0 \leqq \mathrm{~d} \lambda[g<t] / \mathrm{d} \lambda_{\Omega} \leqq 1$ a. e. $\lambda_{\Omega}$. These yield the desired inequality.

Theorem 4.5. Let $\left\{\lambda_{G}: G \in \mathscr{I}_{q}\right\}$ be an arbitrary consistent family of Jensen measures, and let $g \in \mathbb{J}$. Call $h(t, \omega)=\left(\mathrm{d} \lambda[g<t] / \mathrm{d} \lambda_{\Omega}\right)(\omega) \in L^{1}\left(\lambda_{\Omega}\right)$. Then for each $b \in \boldsymbol{R}$, the density $h(t, \omega)$ converges increasingly to $h(b, \omega)$ provided $t$ converges increasingly to $b$, i.e. in $L^{1}\left(\lambda_{\Omega}\right), h(t, \omega) / h(b, \omega)$ as $t / b$. In particular, under the proper modification, the function $R \times \Omega \ni(t, \omega) \mapsto h(t, \omega)$ can be viewed as a measurable function with respect to the product measure $\mathrm{d} t \cdot \mathrm{d} \lambda_{\Omega}$.

Proof. We may assume that $b>g(q)$. Note that for every $t$ with $t \leqq b$, $h(t,) \leqq h(b$,$) a. e. \lambda_{\Omega}$ by Lemma 4.4 . Hence it suffices to show that $\|h(t,)\|_{L 1}$ $=\lambda[g<t](\Omega) \nearrow \lambda[g<b](\Omega)$ as $t / h$, or equivalently $\lim _{t \rightarrow b-0} \lambda[g=t](\Omega)=\lambda[g=b](\Omega)$. Since $\lambda[g<t](\Omega)$ is increasing, $\lambda[g=t](\Omega)=1-\lambda[g<t](\Omega)$ is decreasing with respect to $t$. Consequently we have only to prove

$$
\varlimsup_{t \rightarrow b-0} \lambda[g=t](\Omega) \leqq \lambda[g=b](\Omega) .
$$

Put $Q_{n}=\exp \{n(g-b)\} \quad n \in N$. By Lemma 4.3, $Q_{n}$ is contained in $\mathscr{\nexists}$. In particular, $\int Q_{n} \mathrm{~d} \lambda[g \leqq t] \leqq \int Q_{n} \mathrm{~d} \lambda[g \leqq b]$, because $\lambda[g \leqq t]<\lambda[g \leqq b](t \leqq b)$. For a given positive $\varepsilon$, take $n$ so large that the inequality $\varepsilon>\int Q_{n} \mathrm{~d} \lambda[g<b]$ holds. Then from the fact $\lambda[g \leqq t]=\lambda[g<t]+\lambda[g=t]$, it follows that

$$
\begin{aligned}
\lambda[g=b](\Omega) & \geqq \int Q_{n} \mathrm{~d} \lambda[g \leqq b]-\varepsilon \geqq \int Q_{n} \mathrm{~d} \lambda[g \leqq t]-\varepsilon \\
& \geqq \exp \{n(t-b)\} \lambda[g=t](\Omega)-\varepsilon .
\end{aligned}
$$

Take $t$ so close to $b$ that the inequality $1 \geqq \exp \{n(t-b)\} \geqq 1-\varepsilon$ holds. Then we have $\lambda[g=b](\Omega) \geqq \lambda[g=t](\Omega)-2 \varepsilon$. This yields (4.2), because $\varepsilon$ is arbitrary.

Finally, the latter half of the assertion is a basic result in real analysis, and so we omit the detail.

Definition 4.6. Let $\left\{\lambda_{G}: G \in \mathscr{F}_{q}\right\}$ be an arbitrary consistent family of Jensen measures, and let $\mathbb{J}^{+}=\{g \geqq 0: g \in J\}$. Then for each $g \in \mathbb{J}^{+}$and $p, 0<p<\infty$ we put

$$
M_{B}\left(g^{p}\right)(\omega)=\int_{0}^{\infty} p t^{p-1}\left\{1-\left(\mathrm{d} \lambda[g<t] / \mathrm{d} \lambda_{\Omega}\right)(\omega)\right\} \mathrm{d} t .
$$

The function $M_{B}\left(g^{p}\right) \in L^{1}\left(\lambda_{\Omega}\right)$ will be called the generalized (conditional expectation of) Brownian maximal function of $g^{p}$.

Theorem 4.7. Let $\left\{\lambda_{G}: G \in \mathscr{I}_{q}\right\}$ be a consistent family and let $g \in \mathbb{J}^{+}$. Then for each $p, 0<p<\infty$, 


$$
\int M_{B}\left(g^{p}\right) \mathrm{d} \lambda_{\Omega}=\int_{0}^{\infty} p t^{p-1} \lambda[g=t](\Omega) \mathrm{d} t=-\int_{0}^{\infty} t^{p} \mathrm{~d}(\lambda[g=t](\Omega)) .
$$

Moreover, if $g, g^{p} \in J^{+}$for some $p$ with $0<p<\infty$,

$$
\int_{0}^{\infty} p t^{p-1}\left\{1-\left(\mathrm{d} \lambda[g<t] / \mathrm{d} \lambda_{\Omega}\right)(\omega)\right\} \mathrm{d} t=\int_{0}^{\infty}\left\{1-\left(\mathrm{d} \lambda\left[g^{p}<t\right] / \mathrm{d} \lambda_{\Omega}\right)(\omega)\right\} \mathrm{d} t \quad \text { a.e. } \quad \lambda_{\Omega} .
$$

Proof. Applying Fubini's theorem to (4.3) in Definition 4.6, we see that

$$
\begin{aligned}
\int M_{B}\left(g^{p}\right) \mathrm{d} \lambda_{\Omega} & =\int_{0}^{\infty} p t^{p-1}\{1-\lambda[g<t](\Omega)\} \mathrm{d} t \\
& =\int_{0}^{\infty} p t^{p-1} \lambda[g=t](\Omega) \mathrm{d} t=-\int_{0}^{\infty} t^{p} \mathrm{~d}(\lambda[g=t](\Omega)) .
\end{aligned}
$$

The last expression is due to the fact that the correspondence $R \ni t \rightarrow \lambda[g=t](\Omega)$ gives a left continuous decreasing function on $\boldsymbol{R}$ by Theorem 4.5 .

Next, assume that $g, g^{p} \in J^{+}$. Put $h(t)=,\mathrm{d} \lambda[g<t] / \mathrm{d} \lambda_{\Omega}$ and $k(s)=$, $\mathrm{d} \lambda\left[g^{p}<s\right] / \mathrm{d} \lambda_{\Omega}$. Then for almost all $\omega \in \Omega$ with respect to $\lambda_{\Omega}, 1-h(t)=$, $1-k\left(t^{p},\right) \in L^{1}(0, \infty)$. Therefore

$$
\int_{0}^{\infty}(1-k(t, \omega)) \mathrm{d} t=\int_{0}^{\infty} p t^{p-1}\left(1-k\left(t^{p}, \omega\right)\right) \mathrm{d} t=\int_{0}^{\infty} p t^{p-1}(1-h(t, \omega)) \mathrm{d} t
$$

Finally, we establish two stability theorems for consistent families. The first theorem is almost obvious. So we omit its proof.

Theorem 4.8. Let $\left\{\lambda_{G}: G \in \mathscr{I}_{q}\right\}$ and $\left\{\mu_{G}: G \in \mathscr{F}_{q}\right\}$ be maximally consistent (resp. consistent). Then every convex combination $\left\{s \cdot \lambda_{G}+(1-s) \cdot \mu_{G}: G \in \mathscr{I}_{q}\right\}$, $0 \leqq s \leqq 1$, of them is also maximally consistent (resp. consistent).

Recall that every member of $\mathscr{F}_{q}$ contains $q$ as an interior point. Therefore, if a directed set $\left\{q_{i} \in \Omega: i \in I\right\}$ converges to $q \in \Omega$, then each member $G$ of $\mathscr{F}_{q}$ is contained in $\mathscr{F}_{q_{i}}$ with the index $i$ sufficiently "large".

Theorem 4.9. Let $\left(\left\{\lambda_{G}^{i}: G \in \mathscr{F}_{q_{i}}\right\}\right)_{i \in I}$ be a set of consistent families indexed by a directed set $I$, each of which has base point $q_{i} \in \Omega(i \in I)$. Suppose that $q_{i}$ converges to $q$ on $\Omega$ and that for each $G \in \mathscr{F}_{q}, \lambda_{G}^{i}$ converges weakly-star to $\lambda_{G}$ in the dual of $C(\Omega)$. Then the family $\left\{\lambda_{G}: G \in \mathscr{F}_{q}\right\}$ is consistent.

Proof. It is clear that the family in problem satisfies conditions (1), (2) of Definition 4.1. Let $F, G$ be an arbitrary pair of $\mathscr{F}_{q}$ with $F \subset G$, and let denote by $U$ the relative interior of $F$ with respect to $G$. Take any $g$ of $C_{R}(G)$ that is nonnegative and carried on $U$. Then by the assumption, $\int g \mathrm{~d} \lambda_{F}^{i} \leqq \int g \mathrm{~d} \lambda_{G}^{i}$ with the index $i$ sufficiently "large", so that $\int g \mathrm{~d} \lambda_{F} \leqq \int g \mathrm{~d} \lambda_{G}$. This implies that $0 \leqq \mathrm{~d}\left(\lambda_{F} \mid U\right) / \mathrm{d} \lambda_{G} \leqq 1$ a. e. $\lambda_{G}$. 
Corollary 4.10. Let $E$ be an arbitrary closed subset of $\Omega$ containing the Shilov boundary of $\boldsymbol{A}$, and let $q \in \Omega$. Suppose further that $H_{q}^{E}$ is the totality of Jensen measures for $q$ supported on $E$, each of which is the terminal measure of some consistent family with base point $q$. Then $H_{q}^{E}$ is a weakstar compact convex set in the dual of $C(\Omega)$.

\section{$\S 5$. Burkholder-Gundy-Silverstein Inequalities and Fefferman's Theorem}

In this section, we shall discuss Burkholder-Gundy-Silverstein inequalities and Garsia's definition of BMO. Here we should point out that as far as the former is concerned, our strategy of the proof is analogous to the probabilistic one (cf. [9]).

Lemma 5.1. Let $\left\{\lambda_{G}: G \in \mathscr{F}_{q}\right\}$ be a consistent family, and let $g, f \in \mathbb{J}^{+}$satisfy the inequality $g \leqq f$. Then for each $p$ with $0<p<\infty$,

$$
\int g^{p} \mathrm{~d} \lambda_{\Omega} \leqq \int M_{B}\left(g^{p}\right) \mathrm{d} \lambda_{\Omega} \leqq \int M_{B}\left(f^{p}\right) \mathrm{d} \lambda_{\Omega} .
$$

Proof. For the left-hand inequality, recall that

$$
\int g^{p} \mathrm{~d} \lambda_{\Omega}=\int_{0}^{\infty} p t^{p-1} \lambda_{\Omega}(\Omega[g>t]) \mathrm{d} t \text { and } \int M_{B}\left(g^{p}\right) \mathrm{d} \lambda_{\Omega}=\int_{0}^{\infty} p t^{p-1} \lambda[g=t](\Omega) \mathrm{d} t .
$$

Hence it suffices to show that $\lambda_{\Omega}(\Omega[g>t]) \leqq \lambda[g=t](\Omega)$. Since $\lambda[g=t]+\lambda[g<t]$ $<\lambda_{\Omega}$, we have that $\lambda[g=t]<\lambda_{\Omega}-\lambda[g<t]$. In particular, $\lambda[g=t](\Omega)=$ $\left(\lambda_{\Omega}-\lambda[g<t]\right)(\Omega)$. On the other hand, $\lambda[g<t](\Omega[g>t])=0$, and so

$$
\lambda_{\Omega}(\Omega[g>t])=\left(\lambda_{\Omega}-\lambda[g<t]\right)(\Omega[g>t]) \leqq\left(\lambda_{\Omega}-\lambda[g<t]\right)(\Omega)=\lambda[g=t](\Omega),
$$

because $\lambda_{\Omega}-\lambda[g<t] \geqq 0$ by condition (3) in Definition 4.1 .

For the right-hand inequality, observe that $\Omega[f \leqq t] \subset \Omega[g \leqq t]$. This implies that $\lambda[g=t](\Omega) \leqq \lambda[f=t](\Omega)$. Hence

$$
\int M_{B}\left(g^{p}\right) \mathrm{d} \lambda_{\Omega}=\int_{0}^{\infty} p t^{p-1} \lambda[g=t](\Omega) \mathrm{d} t \leqq \int_{0}^{\infty} p t^{p-1} \lambda[f=t] \mathrm{d} t=\int M_{B}\left(f^{p}\right) \mathrm{d} \lambda_{\Omega} .
$$

Theorem 5.2. (Burkholder-Gundy-Silverstein inequalities [2]) Let $\left\{\lambda_{G}: G \in \mathscr{F}_{q}\right\}$ be an arbitrary consistent family of Jensen measures with base point $q \in \Omega$. Then for each $p$ with $1<p<\infty$, there exists the constant $C_{p}$ dependent only on $p$ such that

$$
\int g^{p} \mathrm{~d} \lambda_{\Omega} \leqq \int M_{B}\left(g^{p}\right) \mathrm{d} \lambda_{\Omega} \leqq C_{p} \int g^{p} \mathrm{~d} \lambda_{\Omega}, \quad g \in \mathbb{J}^{+} .
$$

Moreover, if $f \in A$, then for every $p$ with $0<p<\infty$,

$$
\left.\int ! f\right|^{p} \mathrm{~d} \lambda_{\Omega} \leqq \int M_{B}\left(\left.! f\right|^{p}\right) \mathrm{d} \lambda_{\Omega} \leqq e \int|f|^{p} \mathrm{~d} \lambda_{\Omega} .
$$


Proof. Owing to the preceding lemma, we have only to prove the righthand inequality in each case. We show the first inequality for $p, 0<p<\infty$ under the assumption that $g^{r}$ is a log-envelope function for some $r, 0<r<p$. Observe that with $\alpha=r / p$

$$
\int M_{B}\left(g^{p}\right) \mathrm{d} \lambda_{\Omega}=\int_{0}^{\infty} r t^{r-1} \lambda\left[g=t^{a}\right](\Omega) \mathrm{d} t=-\int_{0}^{\infty} t^{r} \mathrm{~d}\left(\lambda\left[g=t^{a}\right](\Omega)\right) .
$$

For a given positive number $\varepsilon$, take a finite sequence $\left\{t_{k}\right\}_{k=0}^{n}$ of real numbers such that $0=t_{0}<t_{1}<\cdots<t_{n}\left(\|g\|_{\infty}<t_{n}^{\alpha}\right)$ and

$$
-\sum_{k=1}^{n}\left(t_{k}\right)^{r}\left\{\lambda\left[g=t_{k}^{\alpha}\right]-\lambda\left[g=t_{k-1}^{\alpha}\right]\right\}(\Omega)<\varepsilon+\int M_{B}\left(g^{p}\right) \mathrm{d} \lambda_{\Omega} .
$$

Call $\sigma_{k}=\lambda\left[g<t_{k}^{\alpha}\right], 0 \leqq k \leqq n$, for notational convenience. Then the above inequality can be read as

$$
\sum_{k=1}^{n}\left(t_{k}\right)^{r}\left\{\sigma_{k}-\sigma_{k-1}\right\}(\Omega)<\varepsilon+\int M_{B}\left(g^{p}\right) \mathrm{d} \lambda_{\Omega} .
$$

Furthermore, from the facts $\sigma_{0}=0$ and $\sigma_{n}=\lambda_{\Omega}$, it follows that

$$
\lambda_{\Omega}=\sum_{k=1}^{n}\left(\sigma_{k}-\sigma_{k-1}\right) .
$$

Here let us estimate the value $\lambda\left[g=t^{a}\right](\Omega)$. From the relation $\lambda\left[g \leqq t^{a}\right]<\lambda_{\Omega}$, we find that $\lambda\left[g=t^{\alpha}\right]<\lambda_{\Omega}-\lambda\left[g<t^{\alpha}\right]$. Since $g^{r}$ is a log-envelope function, we obtain (in all cases)

$$
t^{a r} \lambda\left[g=t^{a}\right](\Omega) \leqq \int g^{r} \mathrm{~d}\left(\lambda\left[g=t^{a}\right]\right) \leqq \int g^{r} \mathrm{~d}\left(\lambda_{\Omega}-\lambda\left[g<t^{a}\right]\right) .
$$

In particular, if $t_{k-1} \leqq t<t_{k}$, then

$$
\lambda\left[g=t^{\alpha}\right](\Omega) \leqq t^{-a r} \int g^{r} \mathrm{~d}\left(\lambda_{\Omega}-\lambda\left[g<t^{\alpha}\right]\right) \leqq t^{-a r} \int g^{r} \mathrm{~d}\left(\lambda_{\Omega}-\sigma_{k-1}\right)
$$

or by (5.3)

$$
\lambda\left[g=t^{\alpha}\right](\Omega) \leqq t^{-a r} \sum_{j=k}^{n} \int g^{r} \mathrm{~d}\left(\sigma_{j}-\sigma_{j-1}\right)
$$

Let denote by $I_{[t<a]}$ the indicator of the open interval $(-\infty, a)$. Then above inequality can be expressed in the form

$$
\lambda\left[g=t^{a}\right](\Omega) \leqq \sum_{k=1}^{n} t^{-a r} I_{\left[t<t_{k}\right]} \int g^{r} \mathrm{~d}\left(\sigma_{k}-\sigma_{k-1}\right) .
$$

Substituting this inequality in (5.1) and noting that $r=\alpha p$, we have 


$$
\begin{aligned}
& \int M_{B}\left(g^{p}\right) \mathrm{d} \lambda_{\Omega} \leqq \alpha p \int_{0}^{\infty}\left\{\sum_{k=1}^{n} t^{a p-a r-1} I_{\left[t<t_{k}\right]} \int g^{r} \mathrm{~d}\left(\sigma_{k}-\sigma_{k-1}\right)\right\} \mathrm{d} t \\
& =(p / p-r) \sum_{k=1}^{n} \int\left(t_{k}\right)^{a p-a r} g^{r} \mathrm{~d}\left(\sigma_{k}-\sigma_{k-1}\right) \\
& \leqq(p / p-r) \sum_{k=1}^{n}\left\{\left(t_{k}\right)^{r}\left(\sigma_{k}-\sigma_{k-1}\right)(\Omega)\right\}^{(p-r) / p}\left\{\int g^{p} \mathrm{~d}\left(\sigma_{k}-\sigma_{k-1}\right)\right\}^{r / p} \\
& \leqq(p / p-r)\left\{\sum_{k=1}^{n}\left(t_{k}\right)^{r}\left(\sigma_{k}-\sigma_{k-1}\right)(\Omega)\right\}^{(p-r) / p}\left\{\sum_{k=1}^{n} \int g^{p} \mathrm{~d}\left(\sigma_{k}-\sigma_{k-1}\right)\right\}^{r / p} \\
& \quad \leqq(p / p-r)\left\{\varepsilon+\int M_{B}\left(g^{p}\right) \mathrm{d} \lambda_{\Omega}\right\}^{(p-r) / p}\left\{\int g^{p} \mathrm{~d} \lambda_{\Omega}\right\}^{r / p} .
\end{aligned}
$$

The last inequality is due to (5.2), (5.3). Letting $\varepsilon \rightarrow 0$, we obtain

$$
\int M_{B}\left(g^{p}\right) \mathrm{d} \lambda_{\Omega} \leqq(p / p-r)^{p / r} \int g^{p} \mathrm{~d} \lambda_{\Omega} .
$$

This leads us to the final conclusion. Indeed, if $p>1$, we can take 1 as $r$. Thus the first inequality is proved. In case that $g=|f|$, then $g^{r}$ is a logenvelope function for every $r, 0<r<\infty$ by Lemma 4.3. So the second inequality holds.

Lemma 5.3. Let $\left\{\lambda_{G}: G \in \mathscr{F}_{q}\right\}$ be a consistent family, and let $f \in \mathbb{A}$ and $u=\operatorname{Re} f$. Suppose a positive number $\alpha$ satisfies the inequality $\alpha>|f(q)|$. Then for each $\beta \in \boldsymbol{R}$,

$$
\lambda[|f| \leqq \alpha](\Omega[|u| \geqq \beta]) \leqq \lambda[|u|=\beta](\Omega) .
$$

Proof. We may assume that $\beta>|u(q)|$. Note that $|u|=\log \left|e^{f}\right| \vee \log \left|e^{-f}\right|$ belongs to $J_{\text {. }}$ So the set $G=\Omega[|f| \leqq \alpha,|u| \leqq \beta]=\Omega[|f| \leqq \alpha] \cap \Omega[|u| \leqq \beta]$ is contained in $\mathscr{F}_{q}$. We first show that the measure $\lambda_{G}$ of the family has no mass on the set $\Omega[|f|=\alpha,|u|=\beta]$. Indeed, assume that this is false. Then for a suitable complex number $\gamma= \pm \beta \pm i \sqrt{\alpha^{2}-\beta^{2}}$, the inequality $\lambda_{G}(\Omega[f=\gamma])>0$ must hold. Put $g=(f+\gamma) / 2 \gamma$. It is clear that $g \in A,\|g\|_{G}=1=g \mid \Omega[f=\gamma]$, and $|g(q)|<1$, where $q$ is the base point of $\mathscr{F}_{q}$. Hence

$$
0=\lim _{n} g^{n}(q)=\lim _{n} \int g^{n} \mathrm{~d} \lambda_{G}=\lambda_{G}(\Omega[f=\gamma])>0,
$$

a contradiction.

Call $\lambda_{1}=\lambda_{G} \mid \Omega[|f| \leqq \alpha,|u|<\beta]$ and $\lambda_{2}=\lambda_{G} \mid \Omega[|f|<\alpha,|u|=\beta]$. From the above, we find that $\lambda_{G}=\lambda_{1}+\lambda_{2}$. Furthermore, since $\Omega[|f| \leqq \alpha,|u|<\beta]$ is a relatively open subset of $\Omega[|f| \leqq \alpha]$, we are led to the relation $\lambda[|f| \leqq \alpha]-\lambda_{1} \geqq 0$ by condition (3) of Def. 4.1. The similar reason yields that $\lambda[|u| \leqq \beta]-\lambda_{2} \geqq 0$. Therefore we obtain 


$$
\begin{aligned}
& \lambda[|f| \leqq \alpha](\Omega[|u| \geqq \beta])=\left(\lambda[|f| \leqq \alpha]-\lambda_{1}\right)(\Omega[|u| \geqq \beta]) \\
& \leqq\left(\lambda[|f| \leqq \alpha]-\lambda_{1}\right)(\Omega)=1-\lambda_{1}(\Omega)=\lambda_{2}(\Omega)=\lambda_{2}(\Omega[|u|=\beta]) \\
& \leqq \lambda[|u| \leqq \beta](\Omega[|u|=\beta])=\lambda[|u|=\beta](\Omega) .
\end{aligned}
$$

Lemma 5.4. (Paley-Zygmund cf. [9], [10]) Let ע be a positive measure on some measure space. Suppose that for a measurable function $g \geqq 0$, there exist positive numbers $\alpha, \beta$ such that $\alpha\|\nu\| \leqq \int g \mathrm{~d} \nu$ and $\int g^{2} \mathrm{~d} \nu \leqq \beta\|\nu\|$. Then

$$
\nu(\{x: g(x) \geqq \alpha / 2\}) \geqq\|\nu\|(\alpha / 2)^{2} / \beta \text {. }
$$

Theorem 5.5. (Burkholder-Gundy-Silverstein inequalities) Let $\left\{\lambda_{G}: G \in \mathscr{F}_{q}\right\}$ be an arbitrary consistent family of Jensen measures with base point $q \in \Omega$, and let $f=u+i \tilde{u} \in A$, where $u=\operatorname{Re} f$ and $\tilde{u}(q)=0$. Then for each $p, 0<p<\infty$, there exists the universal constant $C_{p}$ such that

$$
\int M_{B}\left(|u|^{p}\right) \mathrm{d} \lambda_{\Omega} \leqq \int M_{B}\left(|f|^{p}\right) \mathrm{d} \lambda_{\Omega} \leqq C_{p} \int M_{B}\left(|u|^{p}\right) \mathrm{d} \lambda_{\Omega} .
$$

Proof. The left-hand inequality is due to Lemma 5.1. For the inequality on the right side, put $I=\left\{t>0: 2^{p+1} \lambda[|f|=2 t](\Omega) \geqq \lambda[|f|=t](\Omega)\right\}$. Here recall the well-known inequality (cf. [9])

$$
\begin{aligned}
& \left(1-\left(2^{p} / 2^{p+1}\right)\right) \int_{0}^{\infty} p t^{p-1} \lambda[|f|=t](\Omega) \mathrm{d} t \\
& \quad \leqq 2^{p}\left(1-2^{-p-1}\right) \int_{I} p t^{p-1} \lambda[|f|=t](\Omega) \mathrm{d} t .
\end{aligned}
$$

Therefore,

$$
\int M_{B}\left(|f|^{p}\right) \mathrm{d} \lambda_{\Omega} \leqq 2^{p+1} \int_{I} p t^{p-1} \lambda[|f|=t](\Omega) \mathrm{d} t .
$$

Let us estimate the value $\lambda[|f|=t](\Omega)$ with $t \in I$. We first verify the case $t>|f(q)|=|u(q)|$. For notational convenience, call $\mu_{1}=\lambda[|f|=t]$ and $\mu_{2}=$ $\lambda[|f| \leqq 2 t]-\lambda[|f|<t]$. By condition (3) in Def. 4.1, $\mu_{2} \geqq 0$ and $\mu_{1} \prec \mu_{2}$. Let $\nu$ be a measure on the product space $\Omega^{2}=\Omega \times \Omega$ that is constructed in Theorem 3.1 for ordered measures $\mu_{1}$ and $\mu_{2}$. Then by $T f=f$ a. e. $\mu_{1}(f \in A)$

$$
\begin{aligned}
& \int(1 \otimes f-f \otimes 1)^{2} \mathrm{~d} \nu=\int\left\{1 \otimes f^{2}+f^{2} \otimes 1-2 f \otimes f\right\} \mathrm{d} \nu \\
& \quad=\int f^{2} \mathrm{~d} \mu_{2}+\int f^{2} \mathrm{~d} \mu_{1}-2 \int f(T f) \mathrm{d} \mu_{1}=\int\left\{T\left(f^{2}\right)+f^{2}-2 f(T f)\right\} \mathrm{d} \mu_{1}=0 .
\end{aligned}
$$

Hence we have that

$$
\int(1 \otimes u-u \otimes 1)^{2} \mathrm{~d} \nu=\int(1 \otimes \tilde{u}-\tilde{u} \otimes 1)^{2} \mathrm{~d} \nu,
$$


so that for $S=\Omega^{2}[|1 \otimes f| \geqq 2 t]$,

$$
\int(1 \otimes u-u \otimes 1)^{2} \mathrm{~d} \nu=2^{-1} \int|1 \otimes f-f \otimes 1|^{2} \mathrm{~d} \nu \geqq 2^{-1} \int_{S} ! 1 \otimes f-\left.f \otimes 1\right|^{2} \mathrm{~d} \nu .
$$

From inequalities $|f \otimes 1|=t,|1 \otimes f| \leqq 2 t$ a. e. $\nu$, the above yields

$$
\begin{aligned}
& \int(1 \otimes u-u \otimes 1)^{2} \mathrm{~d} \nu \geqq 2^{-1} \int_{S}(|1 \otimes f|-|f \otimes 1|)^{2} \mathrm{~d} \nu \\
& \quad=\left(t^{2} / 2\right) \nu\left(\Omega^{2}[|1 \otimes f| \geqq 2 t]\right)=\left(t^{2} / 2\right) \mu_{2}(\Omega[|f| \geqq 2 t])=\left(t^{2} / 2\right) \lambda[|f|=2 t](\Omega) \\
& \quad \geqq t^{2} 2^{-p-2} \lambda[|f|=t](\Omega)=t^{2} 2^{-p-2} \nu\left(\Omega^{2}\right) .
\end{aligned}
$$

On the other hand,

$$
\int(1 \otimes u-u \otimes 1)^{4} \mathrm{~d} \nu \leqq(2 t+t)^{4} \nu\left(\Omega^{2}\right)=(3 t)^{ \pm} \nu\left(\Omega^{2}\right) .
$$

Applying Lemma 5.4 with $g=(1 \otimes u-u \otimes 1)^{2}$, these yield

$$
\nu\left(\Omega^{2}[|1 \otimes u-u \otimes 1| \geqq A t]\right) \geqq B \nu\left(\Omega^{2}\right)=B \lambda[|f|=t](\Omega)
$$

where $A=\left(2^{-p-3}\right)^{1 / 2}$ and $B=\left(2^{-p-3}\right)^{2} / 3^{4}$.

Now, using Lemma 5.3, we are led to the estimate

$$
\begin{aligned}
& \nu\left(\Omega^{2}[|1 \otimes u-u \otimes 1| \geqq A t]\right) \leqq \nu\left(\Omega^{2}[|1 \otimes u| \geqq A t / 2]\right)+\nu\left(\Omega^{2}[|u \otimes 1| \geqq A t / 2]\right) \\
& \quad=\mu_{2}(\Omega[|u| \geqq A t / 2])+\mu_{1}(\Omega[|u| \geqq A t / 2]) \\
& \quad \leqq \lambda[|f| \leqq 2 t](\Omega[|u| \geqq A t / 2])+\lambda[|f| \leqq t](\Omega[|u| \geqq A t / 2]) \\
& \quad \leqq 2 \lambda[|u|=A t / 2](\Omega) .
\end{aligned}
$$

Hence we conclude that

$$
\lambda[|f|=t](\Omega) \leqq(2 / B) \lambda[|u|=A t / 2](\Omega) .
$$

Since $1<2 / B$ and $A / 2<1,(5.5)$ is still valid for the case $t \leqq|f(q)|=|u(q)|$ and $t \in I$. Substituting (5.5) in (5.4), we obtain that

$$
\begin{gathered}
\int M_{B}\left(|f|^{p}\right) \mathrm{d} \lambda_{\Omega} \leqq\left(2^{p+2} / B\right) \int_{I} p t^{p-1} \lambda[|u|=A t / 2](\Omega) \mathrm{d} t \\
\leqq C_{p} \int_{0}^{\infty} p t^{p-1} \lambda[|u|=t](\Omega) \mathrm{d} t=C_{p} \int M_{B}\left(|u|^{p}\right) \mathrm{d} \lambda_{\Omega} .
\end{gathered}
$$

Corollary 5.6. (Burkholder-Gundy-Silverstein) Let $\left\{\lambda_{G}: G \in \mathscr{F}_{q}\right\}$ be a consistent family, and let $f=u+i \tilde{u} \in \mathbb{A}$ with $\tilde{u}(q)=0$. Then for each $p, 0<p<\infty$, there exists the universal constant $C_{p}$ such that $\int M_{B}\left(|\tilde{u}|^{p}\right) \mathrm{d} \lambda_{\Omega} \leqq C_{p} \int M_{B}\left(|u|^{p}\right) \mathrm{d} \lambda_{\Omega}$.

In the remainder of this section, we discuss Fefferman's duality theorem on BMO [4]. Let $h$ be an (abstract) harmonic function on $\Omega$. Namely, $h$ is a 
real-valued continuous function on $\Omega$ such that $h=\check{h}=\hat{h}$, where $\hat{h}=-(-h)$. Note that every function of $\operatorname{Re} A$ is harmonic on $\Omega$. According to the original definition of Garsia (semi-) norm, we put, for any harmonic function $h$, (cf. [8])

$$
\eta(h)=\left\{\sup \left\{\left(\widehat{h^{2}}-h^{2}\right)(\omega): \omega \in \Omega\right\}\right\}^{1 / 2} .
$$

Let $\mu_{1}, \mu_{2}$ be positive measures on $\Omega$ with $\mu_{1} \prec \mu_{2}$, and let $T: L^{p}\left(\mu_{2}\right) \rightarrow L^{p}\left(\mu_{1}\right)$, $1 \leqq p \leqq \infty$, be an arbitrary conditional expectation. By condition (3) in Theorem 3.1, the inequality $T g \geqq g$ a. e. $\mu_{1}$ is valid for $g \in \boldsymbol{J}$ and so, for every log-envelope function on $\Omega$. In particular $T g=g$ a.e. $\mu_{1}$, whenever $g$ is harmonic on $\Omega$, or $g \in A$. Furthermore, if $h$ is a harmonic function on $\Omega$, then $0 \leqq T\left(h^{2}\right)-h^{2}$ $\leqq\{\eta(h)\}^{2}$ a. e. $\mu_{1}$, because $h^{2} \leqq T\left(h^{2}\right) \leqq T\left(\widehat{h^{2}}\right) \leqq \widehat{h^{2}}$ a. e. $\mu_{1}$.

Theorem 5.7. Let $\left\{\lambda_{G}: G \in \mathscr{F}_{q}\right\}$ be an arbitrary consistent family of Jensen measures and let $h$ be a harmonic function on $\Omega$. Then for each $f \in \boldsymbol{A}$ with $f(q)=0$,

$$
\left|\int f h \mathrm{~d} \lambda_{\Omega}\right| \leqq e \sqrt{2} \eta(h) \int|f| \mathrm{d} \lambda_{\Omega}
$$

Proof. Given a positive number $\varepsilon$, we take a finite sequence $\left\{t_{k}\right\}_{k=0}^{n}$ of real numbers such that $0=t_{0}<t_{1}<\cdots<t_{n}\left(\|f\|<t_{n}\right)$ and

$$
\sum_{k=1}^{n}\left(t_{k}-t_{k-1}\right) \lambda\left[|f|=t_{k-1}\right](\Omega)<\varepsilon+\int M_{B}(|f|) \mathrm{d} \lambda_{\Omega} .
$$

Let $\nu_{k}$ be a measure on $\Omega^{2}=\Omega \times \Omega$ constructed in Theorem 3.1 with $\mu_{1}=$ $\lambda\left[|f| \leqq t_{k-1}\right]$ and $\mu_{2}=\lambda\left[|f| \leqq t_{k}\right], \quad 1 \leqq k \leqq n$. Then noting that $f(q)=0$ and $\lambda[|f| \leqq 0]=\delta_{q}$, we have

$$
\begin{aligned}
\int h f \mathrm{~d} \lambda_{\Omega} & =\sum_{k=1}^{n} \int h f \mathrm{~d}\left(\lambda\left[|f| \leqq t_{k}\right]-\lambda\left[|f| \leqq t_{k-1}\right]\right) \\
& =\sum_{k=1}^{n} \int(1 \otimes h f-h f \otimes 1) \mathrm{d} \nu_{k} .
\end{aligned}
$$

On the other hand,

$$
E(h \otimes f \mid \sigma\{C(\Omega) \otimes 1\})=E(f \otimes h \mid \sigma\{C(\Omega) \otimes 1\})=h f \otimes 1 \quad \text { a. e. } \quad \nu_{k}(1 \leqq k \leqq n) .
$$

So, the above yields

$$
\int h f \mathrm{~d} \lambda_{\Omega}=\sum_{k=1}^{n} \int(1 \otimes f-f \otimes 1)(1 \otimes h-h \otimes 1) \mathrm{d} \nu_{k} .
$$

Therefore

$$
\left|\int h f \mathrm{~d} \lambda_{\Omega}\right| \leqq \sum_{k=1}^{n}\left(\int \frac{|1 \otimes f-f \otimes 1|^{2}}{t_{k}} \mathrm{~d} \nu_{k}\right)^{1 / 2}\left(\int t_{k}|1 \otimes h-h \otimes 1|^{2} \mathrm{~d} \nu_{k}\right)^{1 / 2} .
$$

Here observe that 


$$
\begin{aligned}
& \int|1 \otimes f-f \otimes 1|^{2} \mathrm{~d} \nu_{k}=\int|f|^{2} \mathrm{~d} \lambda\left[|f| \leqq t_{k}\right]-\int|f|^{2} \mathrm{~d} \lambda\left[|f| \leqq t_{k-1}\right] \\
& \quad=\int|f|^{2} \mathrm{~d}\left(\lambda\left[|f|<t_{k}\right]-\lambda\left[|f|<t_{k-1}\right]\right)+t_{k}^{2} \lambda\left[|f|=t_{k}\right](\Omega)-t_{k-1}^{2} \lambda\left[|f|=t_{k-1}\right](\Omega) \\
& \quad \leqq t_{k}^{2}\left(\lambda\left[|f|<t_{k}\right]-\lambda\left[\left|f_{\mid}\right|<t_{k-1}\right]\right)(\Omega)+t_{k}^{2} \lambda\left[|f|=t_{k}\right](\Omega)-t_{k-1}^{2} \lambda\left[|f|=t_{k-1}\right](\Omega) \\
& =\left(t_{k}^{2}-t_{k-1}^{2}\right) \lambda\left[|f|=t_{k-1}\right](\Omega) .
\end{aligned}
$$

Hence

$$
\int \frac{|1 \otimes f-f \otimes 1|^{2}}{t_{k}} \mathrm{~d} \nu_{k} \leqq 2\left(t_{k}-t_{k-1}\right) \lambda\left[|f|=t_{k-1}\right](\Omega) .
$$

Also we find that

$$
\int t_{k}|1 \otimes h-h \otimes 1|^{2} \mathrm{~d} \nu_{k}=t_{k}\left(\int h^{2} \mathrm{~d} \lambda\left[|f| \leqq t_{k}\right]-\int h^{2} \mathrm{~d} \lambda\left[|f| \leqq t_{k-1}\right]\right) .
$$

From these, and by (5.6), it follows that

$$
\begin{aligned}
& \left|\int h f \mathrm{~d} \lambda_{\Omega}\right| \leqq \sum_{k=1}^{n}\left\{2\left(t_{k}-t_{k-1}\right) \lambda\left[|f|=t_{k-1}\right](\Omega)\right\}^{1 / 2} \\
& \quad \times\left\{t_{k}\left(\int h^{2} \mathrm{~d} \lambda\left[|f| \leqq t_{k}\right]-\int h^{2} \mathrm{~d} \lambda\left[|f| \leqq t_{k-1}\right]\right)\right\}^{1 / 2} \\
& \leqq\left\{2 \sum_{k=1}^{n}\left(t_{k}-t_{k-1}\right) \lambda\left[|f|=t_{k-1}\right](\Omega)\right\}^{1 / 2} \\
& \quad \times\left\{\sum_{k=1}^{n} t_{k}\left(\int h^{2} \mathrm{~d} \lambda\left[|f| \leqq t_{k}\right]-\int h^{2} \mathrm{~d} \lambda\left[|f| \leqq t_{k-1}\right]\right)\right\}^{1 / 2} \\
& \leqq\left\{2 \varepsilon+2 \int M_{B}(|f|) \mathrm{d} \lambda_{\Omega}\right\}^{1 / 2} \\
& \times\left\{\sum_{k=1}^{n}\left(t_{k}-t_{k-1}\right)\left(\int h^{2} \mathrm{~d} \lambda_{\Omega}-\int h^{2} \mathrm{~d} \lambda\left[|f| \leqq t_{k-1}\right]\right)\right\}^{1 / 2}
\end{aligned}
$$

On the other hand, the following equality holds:

$$
\int h^{2} \mathrm{~d} \lambda_{\Omega}-\int h^{2} \mathrm{~d} \lambda\left[|f| \leqq t_{k-1}\right]=\int h^{2} \mathrm{~d}\left(\lambda_{\Omega}-\lambda\left[|f|<t_{k-1}\right]\right)-\int h^{2} \mathrm{~d} \lambda\left[|f|=t_{k-1}\right],
$$

and also we have $\lambda\left[|f|=t_{k-1}\right]<\lambda_{\Omega}-\lambda\left[|f|<t_{k-1}\right]$. Let $T$ be any conditional expectation between $\lambda\left[|f|=t_{k-1}\right]$ and $\lambda_{\Omega}-\lambda\left[|f|<t_{k-1}\right]$. Then as pointed out earlier, $0 \leqq T\left(h^{2}\right)-h^{2} \leqq \eta(h)^{2}$ a. e. $\lambda\left[|f|=t_{k-1}\right]$. Hence

$$
\begin{aligned}
\int h^{2} \mathrm{~d} \lambda_{\Omega}-\int h^{2} \mathrm{~d} \lambda\left[|f| \leqq t_{k-1}\right] & =\int\left(T\left(h^{2}\right)-h^{2}\right) \mathrm{d} \lambda\left[|f|=t_{k-1}\right] \\
& \leqq\{\eta(h)\}^{2} \lambda\left[|f|=t_{k-1}\right](\Omega)
\end{aligned}
$$

Substituting this inequality in (5.7), we obtain by (5.6) that 


$$
\begin{aligned}
\left|\int h f \mathrm{~d} \lambda_{\Omega}\right| & \leqq\left\{2 \varepsilon+2 \int M_{B}(|f|) \mathrm{d} \lambda_{\Omega}\right\}^{1 / 2}\left\{\eta(h)^{2} \sum_{k=1}^{n}\left(t_{k}-t_{k-1}\right) \lambda\left[|f|=t_{k-1}\right](\Omega)\right\}^{1 / 2} \\
& \leqq \sqrt{2} \eta(h)\left(\varepsilon+\int M_{B}(|f|) \mathrm{d} \lambda_{\Omega}\right) .
\end{aligned}
$$

Letting $\varepsilon \rightarrow 0$, and noting Theorem 5.2 , we conclude that

$$
\left|\int h f \mathrm{~d} \lambda_{\Omega}\right| \leqq \sqrt{2} \eta(h) \int M_{B}(|f|) \mathrm{d} \lambda_{\Omega} \leqq e \sqrt{2} \eta(h) \int|f| \mathrm{d} \lambda_{\Omega} .
$$

\section{$\S 6$. The Algebra $R(K)$}

Throughout this section, we shall deal with the concrete algebra $\boldsymbol{R}(K)$, which has been introduced in Section 0. It is known that the maximal ideal space of $\boldsymbol{R}(K)$ is identical with $K$ including the topology. We are interested in the case that $K$ has nonempty interior.

Let $G$ be a compact plane set with an interior point $q$. We denote by $\omega_{q}^{G}$ (resp. $\kappa_{q}^{G}$ ) the harmonic measure (resp. Keldysh measure) for $q$ associated with the set $G$. From Wiener's construction of $\omega_{q}^{G}$ and $\kappa_{q}^{G}$, it is easily seen that the relation $\omega_{q}^{F} \prec \omega_{q}^{G}, \kappa_{q}^{F} \prec \kappa_{q}^{G}$ is valid under the assumption $F \subset G \subset K$. Their extremal properties among Jensen measures were first observed by A. Debiard and B. Gaveau [3].

Lemma 6.1. Let $\boldsymbol{A}=\boldsymbol{R}(K)$ and $q \in \operatorname{Int} K$. Suppose $G$ is a compact subset of $\Omega=K$ whose interior contains $q$. Then the harmonic measure $\omega_{q}^{G}$ is the minimum element in the logarithmic order among all Jensen measures for $q$ that are supported on $K \backslash \operatorname{Int} G$. Furthermore, the Keldysh measure $\kappa_{q}^{G}$ for $q$ is the maximum element in the logarithmic order among all Jensen measures for $q$ that are supported on $G$, if $G$ is $\boldsymbol{R}(K)$-convex.

Lemma 6.2. Let $\boldsymbol{A}=\boldsymbol{R}(K)$ and $q \in \operatorname{Int} K$. Let $\mu$ be an arbitrary Jensen measure for $q$. Then for each decomposition $\mu=\mu_{1}+\mu_{2}$ with $0 \leqq \mu_{1}, \mu_{2}$, the corresponding decomposition $\kappa_{q}^{\Omega}=\kappa_{1}+\kappa_{2}$ with $0 \leqq \kappa_{j}$ and $\mu_{j}<\kappa_{j}(j=1,2)$ is unique.

Proof. The existence of corresponding decompositions is due to CartierFell-Meyer theorem (cf. [1].) Let $\kappa_{q}^{\Omega}=\tilde{\kappa}_{1}+\tilde{\kappa}_{2}$ be another decomposition as above. Then $\kappa_{1}+\tilde{\kappa}_{2}$ is a Jensen measure for $q$ maximal in the logarithmic order. Hence $\kappa_{q}^{\Omega}=\kappa_{1}+\tilde{\kappa}_{2}$, by Lemma 6.1, and accordingly $\kappa_{2}=\tilde{\kappa}_{2}$. (Note that this lemma is covered by general Choquet theorem, cf. [1], [3].)

Lemma 6.3. Let $\boldsymbol{A}=\boldsymbol{R}(K)$ and let $q \in \operatorname{Int} K$. Suppose that $\omega_{q}^{K}=\kappa_{q}^{K}$. Then for every $G \in \mathscr{F}_{q}, \omega_{q}^{G}=\kappa_{q}^{G}$.

Proof. Recall that each $G \in \mathscr{F}_{q}$ is defined as $G=\{z \in K: h(z) \leqq \gamma\}$, where $r \in \boldsymbol{R}$ and $h \in \boldsymbol{J}$. Since each $h \in \boldsymbol{J}$ is subharmonic on Int $K$, every connected 
component of Int $K \backslash G$ is not relatively compact in Int $K$. Hence by Gonchar's criterion, each $z_{0}$ of $\partial G \cap$ Int $K$ is a peak point for $\mathbb{R}(G)$. In particular, $z_{0}$ belongs to the Jensen boundary of $\boldsymbol{R}(G)$. On the other hand, $\mathbb{R}(G)$ is identical with $A_{G}$. Indeed, for each $c \in C \backslash G,(z-c)^{-1} \mid G$ is uniformly approximated by functions in $\boldsymbol{R}(K) ! G$, where $z$ denotes the coordinate function on the complex plane. This is due to the fact that $G$ is $\mathbb{R}(K)$-convex. From this fact, we easily obtain that $\boldsymbol{A}_{G}=\boldsymbol{R}(G)$.

Assume that $\omega_{q}^{K}=\kappa_{q}^{K}$. Given an arbitrary element $G$ of $\mathscr{I}_{q}$, we put $\mu_{1}=\omega_{q}^{G} \mid($ Int $K)$ and $\mu_{2}=\omega_{q}^{G} \mid \partial K$. From the above consideration, $\mu_{1}$ is maximal on G. Let $\omega_{q}^{K}=\omega_{1}+\omega_{2}$ be the decomposition of $\omega_{q}^{K}$ such that $0 \leqq \omega_{j}$ and $\mu_{j} \prec \omega_{j}$ $(j=1,2)$. Then $\omega_{1}+\mu_{2}$ is a Jensen measure for $q$ supported on $\partial K$. Therefore $\omega_{q}^{K}<\omega_{1}+\mu_{2}$ by Lemma 6.1. Since $\omega_{q}^{K}$ is maximal, we find that $\omega_{q}^{K}=\omega_{1}+\mu_{2}$, so that $\mu_{2}=\omega_{2}$. This implies that $\mu_{2}$ is maximal on $\Omega$ in the logarithmic order. Thus we conclude that $\omega_{q}^{G}=\mu_{1}+\mu_{2}$ is maximal on $G$.

Proposition 6.4. Let $\boldsymbol{A}=\boldsymbol{R}(K)$ and $q \in \operatorname{Int} K$. Then the family $\left\{\kappa_{q}^{G}: G \in \mathscr{F}_{q}\right\}$ of Keldysh measures is maximally consistent, and the family $\left\{\omega_{q}^{G}: G \in \mathscr{F}_{q}\right\}$ of harmonic measures is consistent.

Proof. The first assertion is a corollary of Lemma 6.1. For the latter half of the assertion, it suffices to verify condition (3) of Definition 4.1. Let $F$, $G$ be any elements of $\mathscr{F}_{q}$ with $F \subset G$. Put $V=F \cap \operatorname{Int} G$, and let denote by $U$ the relative interior of $F$ with respect to $G$. Note that $V \cap U \subset \operatorname{Int} F$, so that $\omega_{q}^{F}(V \cap U)=0$. For the decomposition $\omega_{q}^{F}=\left(\omega_{q}^{F}-\omega_{q}^{F} \mid V\right)+\omega_{q}^{F} \mid V$, there exists a decomposition $\omega_{q}^{G}=\omega_{1}+\omega_{2}$ such that $0 \leqq \omega_{1}, \omega_{2}$ and $\omega_{q}^{F}-\omega_{q}^{F}\left|V \prec \omega_{1}, \omega_{q}^{F}\right| V \prec \omega_{2}$. Then the measure $\omega_{3}=\omega_{2}+\omega_{q}^{F}-\omega_{q}^{F} \mid V$ is supported on $K \backslash$ Int $G$ and satisfies the relation $\omega_{3} \prec \omega_{q}^{G}$. These imply that $\omega_{3}=\omega_{q}^{G}$ by Lemma 6.1. Hence $\omega_{q}^{G} \mid U=$ $\omega_{3}\left|U \geqq \omega_{q}^{F}\right| U$, because of the fact $\omega_{q}^{F}(V \cap U)=0$. Consequently we obtain that $0 \leqq \mathrm{~d}\left(\omega_{q}^{F} \mid U\right) / \mathrm{d} \omega_{q}^{G} \leqq 1$ a. e. $\omega_{q}^{G}$.

Corollary 6.5. Let $\boldsymbol{A}=\boldsymbol{R}(K)$ and $q \in \operatorname{Int} K$. Then consistent families with base point $q$ whose terminal measures are supported on $\partial K$ are unique if and only if $\omega_{q}^{\Omega}=\kappa_{q}^{\Omega}, \Omega=K$.

Proof. One direction is clear by Proposition 6.4. Let $\left\{\lambda_{G}: G \in \mathscr{F}_{q}\right\}$ be a consistent family with $\lambda_{\Omega}=\omega_{q}^{\Omega}=\kappa_{q}^{\Omega}$. Owing to Lemma 6.3 , we have only to prove that $\lambda_{G}$ (Int $\left.G\right)=0$. But this is an immediate consequence from the facts $\lambda_{G} \mid$ Int $G \leqq \omega_{q}^{\Omega}$ and $\omega_{q}^{\Omega}($ Int $K)=0$.

Remark 6.6. Let $\boldsymbol{A}=\boldsymbol{R}(K)$ and $q \in \operatorname{Int} K$. Here let us agree to denote by $H_{q}^{\partial K}$ the class of Jensen measures for $q$, each of which is supported on $\partial K$ and identical with the terminal measure of some consistent family. From stability theorems, $H_{q}^{\partial K}$ is a weak-star compact convex set in the dual of $\left.\boldsymbol{C}_{(}^{\prime} K\right)$. Of 
course, $H_{q}^{\partial K}$ contains both of the harmonic measure and Keldysh measure. $H_{q}^{\partial K}$ is a one-point set if and only if $\omega_{q}^{\Omega}=\kappa_{q}^{\Omega}$.

In case that $\omega_{q}^{\Omega} \neq \kappa_{q}^{\Omega}$, we can give a Jensen measure of $H_{q}^{\partial K}$ which is different from the convex combination of $\omega_{q}^{\Omega}$ and $\kappa_{q}^{\Omega}$. Let $h$ be any function of $L^{\infty}\left(\omega_{q}^{\Omega}\right)$ with $0 \leqq h \leqq 1$. Using this function $h$, we decompose each $\omega_{q}^{G}, G \in \mathscr{F}_{q}$, into two pieces $\omega_{q}^{G}=\omega_{1}^{G}+\omega_{2}^{G}$. They are defined by $\mathrm{d} \omega_{1}^{G}=h \mathrm{~d}\left(\omega_{q}^{G} \mid V_{G}\right)$ and $\omega_{2}^{G}=\omega_{q}^{G}-\omega_{1}^{G}$, where $V_{G}$ denotes the relative interior of $G$ with respect to $K$. Since $\omega_{q}^{G} \mid V_{G}$ is absolutely continuous with respect to $\omega_{q}^{\Omega}, \omega_{1}^{G}$ is well-defined. We sweep out the measure $\omega_{1}^{G}$ onto the Jensen boundary of $\boldsymbol{A}_{G}=\boldsymbol{R}(G)$ to obtain the measure $\kappa_{1}^{G}$, i. e. $0 \leqq \kappa_{q}^{G}-\kappa_{1}^{G}$ and $\omega_{1}^{G} \prec \kappa_{1}^{G}$. By Lemma 6.2, such balayage of the mass is unique. Put $\lambda_{G}=\kappa_{1}^{G}+\omega_{2}^{G}$. We show that the family $\left\{\lambda_{G}: G \in \mathscr{F}_{q}\right\}$ is consistent. It is clear that every $\lambda_{G}$ is a Jensen measure for $q$. Next, let $F, G \in \mathscr{F}_{q}$ satisfy the relation $F \subset G$. Then from the fact $V_{F} \subset V_{G}$, it follows that $\omega_{q}^{F}\left|V_{F} \leqq \omega_{q}^{G}\right| V_{G}$, so that $\omega_{1}^{F} \leqq \omega_{1}^{G}$. Let $\kappa_{1}^{G}=\kappa_{11}^{G}+\kappa_{12}^{G}$ be the decomposition of $\kappa_{1}^{G}$ such that $\omega_{1}^{F}<\kappa_{11}^{G}$ and $\omega_{1}^{G}-\omega_{1}^{F} \prec \kappa_{12}^{G}$. By the uniqueness of balayage, we find that $\kappa_{1}^{F} \prec \kappa_{11}^{G}$. Hence, $\omega_{2}^{F}<\omega_{q}^{G}-\omega_{1}^{F} \prec \omega_{2}^{G}+\kappa_{12}^{G}$ and $\omega_{2}^{F}+\kappa_{1}^{F} \prec \omega_{2}^{G}+\kappa_{12}^{G}+\kappa_{11}^{G}$, i. e. $\lambda_{F} \prec \lambda_{G}$. Furthermore, if $U$ is the relative interior of $F$ with respect to $G$, and if $g$ is a nonnegative continuous function supported on $U$, then by $V_{F}=U \cap V_{G}$

$$
\begin{aligned}
\int g \mathrm{~d} \lambda_{F} & =\int_{V_{F}} g \mathrm{~d} \lambda_{F}+\int_{U \backslash V_{F}} g \mathrm{~d} \lambda_{F} \\
& =\int_{U} g \mathrm{~d} \kappa_{1}^{F}+\int_{V_{F}} g(1-h) \mathrm{d} \omega_{q}^{F}+\int_{U \backslash V_{F}} g \mathrm{~d} \omega_{q}^{F} \\
& \leqq \int_{U} g \mathrm{~d} \kappa_{11}^{G}+\int_{U} g \mathrm{~d} \kappa_{12}^{G}+\int_{V_{G}} g(1-h) \mathrm{d} \omega_{q}^{G}+\int_{U \backslash V_{G}} g \mathrm{~d} \omega_{q}^{G},
\end{aligned}
$$

because $\kappa_{1}^{F} \mid U \leqq \kappa_{11}^{G}$ by Theorem 2.4, and $\omega_{q}^{F} \mid U \leqq \omega_{q}^{G}$ by Proposition 6.4. This yields the inequality $\int g \mathrm{~d} \lambda_{F} \leqq \int g \mathrm{~d} \lambda_{G}$. Since $g$ is arbitrary, we conclude that $0 \leqq \mathrm{~d}\left(\lambda_{F} \mid U\right) / \mathrm{d} \lambda_{G} \leqq 1$ a. e. $\lambda_{G}$.

Thus our family of Jensen measures is surely consistent. It is clear that for suitable choice of $h$, the resulting family gives the desired example: in the present situation, the harmonic measure is not absolutely continuous with respect to the Keldysh measure. In particular, $H_{q}^{\partial K}$ is infinite dimensional.

Finally, from several reasons, we pose here an open problem. Does the class $H_{q}^{\partial K}$ contain all Jensen measures that are carried on $\partial K$ ?

\section{$\S 7$. Some Remarks}

On the algebras $H^{\infty}(D)$, some comments should be made, where $H^{\infty}(D)$ denotes the Banach algebra of all bounded analytic functions on a given domain $D$ in the complex plane.

We start by summarizing some properties of $H^{\infty}(D)$ common with $\mathbb{R}(K)$. 
Recall that the maximal ideal space $\Omega$ of $H^{\infty}(D)$ contains $D$ as an open subset. Let $q \in D$ and $G \in \mathscr{F}_{q^{\circ}}$. It is known that for every such $G$, the set $G \backslash \operatorname{Int}(D \cap G)$ carries the harmonic measure $\omega_{q}^{G}$ for $q$. The measure $\omega_{q}^{G}$ has a certain minimality property with respect to the logarithmic order. This is a dual version of the fact that for each $h \in C_{R}(\Omega \backslash \operatorname{Int}(D \cap G)), \quad \check{h}$ is harmonic on $\operatorname{Int}(D \cap G)$. About the behavior of such functions, the details can be found in [6].

Lemma 7.1. (T. Gamelin, cf. [6]) Let $A=H^{\infty}(D)$ and $q \in D$. Then the harmonic measure $\omega_{q}^{G}, G \in \mathscr{F}_{q}$, is the minimum element in the logarithmic order among all Jensen measures for $q$ that are supported on $\Omega \backslash \operatorname{Int}(D \cap G)$.

From this lemma and by the argument as in Proposition 6.4, the next observation is immediate.

Proposition 7.2. Let $\boldsymbol{A}=H^{\infty}(D)$ and $q \in D$. Then the family $\left\{\omega_{q}^{G}: G \in \mathscr{F}_{q}\right\}$ of harmonic measures are consistent.

The following is the analogue of Lemma 6.3. Since the argument is strictly same, we omit its proof.

Proposition 7.3. Let $A=H^{\infty}(D)$ and $q \in D$. Suppose the Jensen measure for $q$ supported on $\Omega \backslash D$ is unique. Then for each $G \in \mathscr{F}_{q}$, the Jensen measure for $q$ supported on $G \backslash \operatorname{Int}(D \cap G)$ is unique.

Remark 7.4. The above is the case with the algebra $H^{\infty}(\Delta), \Delta$ the unit disk on $C$. Indeed, let $h$ be a function of $\mathbb{C}_{R}(\Omega \backslash \Delta)$. Then the functions $\check{h}$ and $\hat{h}=-(-h)$ are harmonic on $\Delta$, and extend continuously to the Shilov boundary of $H^{\infty}(\Delta)$. Hence we see that $\check{h}=\hat{h}$ on $\Delta$. This implies that the Jensen measure for $q \in \Delta$ carried on $\Omega \backslash \Delta$ is unique, i. e. only $\omega_{q}^{\Omega}$.

\section{References}

[1] Alfsen, E. M., Compact convex sets and boundary integrals, Springer-Verlag, Berlin, 1971.

[2] Burkholder, D.L., Gundy, R.F. and Silverstein, M.L., A maximal function characterization of the class $H^{p}$, Trans. Amer. Math. Soc., 157 (1971), 137-153.

[3] Debiard, A. and Gaveau, B., Potential fin et algebras de fonctions analytiques I, $J$. Functional analysis, 16 (1974), 289-304.

[4] Fefferman, C. and Stein, E. M., $H^{p}$ spaces of several variables, Acta Math., 129 (1972), 137-197.

[5] Gamelin, T.W., Uniform algebras, Prentice-Hall, Engliwood Cliffs, 1969.

[6] - Uniform algebras and Jensen measures, London Math. Soc. Lect. Notes Series 32, Cambridge University Press, Cambridge, 1978.

[7] Gamelin, T.W. and Sibony, N., Subharmonicity for uniform algebras, J. Functional analysis, 35 (1980), 64-108.

[8] Garnett, J.B., Bounded analytic functions, Academic Press, New York, 1981. 
[9] Petersen, K.E., Brownian motion, Hardy spaces and bounded mean oscillation, London Math. Soc. Lect. Notes Series, 28, Cambridge University Press, Cambridge, 1977.

[10] Zygmund, A., Trigonometric series, I and II, Cambridge University Press, Cambridge, 1959. 